\title{
A proposal for a quality control protocol in breast CT with synchrotron radiation
}

\author{
Adriano Contillo', Anna Veronese 2 , Luca Brombal², Sandro Donato², Luigi Rigon², \\ Angelo Taibi ${ }^{1}$, Giuliana Tromba ${ }^{3}$, Renata Longo ${ }^{2}$, Fulvia Arfelli²
}

\author{
1 University of Ferrara and INFN section of Ferrara, Italy \\ 2 University of Trieste and INFN section of Trieste, Italy \\ ${ }^{3}$ Elettra-Sincrotrone Trieste S.C.p.A., Italy
}

Radiol Oncol 2018; 52(3): 329-336.

Received: 4 December 2017

Accepted: 12 December 2017

Correspondence to: Dr. Adriano Contillo, Università degli Studi di Ferrara, Dipartimento di Fisica e Scienze della Terra, Via Saragat 1 , 44122 Ferrara, Italy. Phone: +390532974235; Fax: +390532974210; E-mail: contillo@fe.infn.it

Disclosure: No potential conflicts of interest were disclosed.

Background. The SYRMA-3D collaboration is setting up the first clinical trial of phase-contrast breast CT with synchrotron radiation at the Elettra synchrotron facility in Trieste, Italy. In this communication, a quality control protocol for breast CT is proposed, and a first test of image quality measurements is performed by means of a custom-made radiographic phantom.

Materials and methods. A set of projections is acquired and used to perform a CT reconstruction of two selected portions of the phantom. Such portions contain a uniform layer of water and a set of radiographic inserts, respectively. Together, they allow to perform several image quality measurements, namely CT number linearity, reconstruction accuracy, uniformity, noise, and low contrast resolution. All measurements are repeated at different beam energies in the range of interest, and at two different dose values.

Results. Measurements show a good linearity in the soft tissue range, paired to a high accuracy of the CT number reconstruction. Uniformity and noise measurements show that reconstruction inhomogeneities are bound to a few percent of the average pixel values. However, low contrast detectability is limited to the higher portion of the explored energy range.

Conclusions. The results of the measurements are satisfactory in terms of their quality, feasibility and reproducibility. With minimal modifications, the phantom is promising to allow a set of image quality measurements to be used in the upcoming clinical trial.

Key words: breast CT; quality control; radiographic phantom; synchrotron radiation

\section{Introduction}

Breast cancer is among the most frequently diagnosed cancers and one of the leading causes of death for women worldwide. Unfortunately, the complex pattern of structures composing the breast parenchyma can significantly reduce the visibility of interesting details, especially in dense breasts. Such effect can hinder early detection, which is a key factor in treating and defeating breast tumors. For this reason, researchers in the field carried out a significant effort during the last decades in investigating 3D imaging modalities, in particular breast computed tomography (CT). In fact, thanks to its intrinsic decoupling of overlapping structures into parallel planes, CT imaging is the most natural answer to any issue related to anatomical noise, although it is worth mentioning other imaging techniques, like ultrasound and magnetic resonance, as valuable complementary techniques routinely used for breast cancer detection.

The design of breast CT, which allows a complete $3 \mathrm{D}$ reconstruction of the uncompressed organ, was theorized several decades ago. ${ }^{1}$ However, recent technological advances favored a strong revival of the field, starting from a feasibility study ${ }^{2}$ which 
revealed the potential for high signal-to-noise ratio images with low anatomic noise, obtainable at dose levels comparable to those for mammography. Several research groups worldwide are developing breast $\mathrm{CT}$ prototypes. Among these groups it is worth mentioning the Friedrich-Alexander University of Erlangen-Nürnberg (Germany) ${ }^{3}$ and the Rochester Medical Center (USA), that promoted a startup company to manufacture and commercialize their prototype. ${ }^{4}$ The SYRMA-3D (synchrotron radiation for mammography) collaboration is in the process of setting up the first clinical trial of phase-contrast breast CT with synchrotron radiation at the SYRMEP (synchrotron radiation for medical physics) beamline of the Elettra synchrotron facility in Trieste..$^{5-8}$ One of the most peculiar features of a synchrotron facility is the high coherence of the X-ray beam, which allows to access the diagnostic information encrypted in its phase profile. ${ }^{9}$ Radiation-matter interaction phenomena involving phase shift are most sensitive to a certain range of imaging details, and such range is distinct from the one of the phenomena involving attenuation. Therefore, accessing the phase profile information is likely to improve the diagnostic potential of the device. Moreover, being tunable to a highly monochromatic beam, synchrotron radiation delivers a lower radiation dose to the imaged organ with respect to conventional X-ray sources, as showed for example in ${ }^{10,11}$. These properties concur in making the SYRMA-3D a unique set up for clinical breast CT examinations.

In order to open a clinical trial, it is first necessary to develop and implement a complete Quality Control (QC) protocol. The enforcement of a QC protocol is paramount to provide the best image quality and the highest patient safety, and to guarantee that said characteristics are preserved over the lifetime of the imaging device. A complete protocol proposal was elaborated by the SYRMA-3D collaboration. ${ }^{12}$ It consists of a list of pre-patient tests, alignment checks, dose and image quality measurements, supplemented with precise directions to the associated measuring procedures. Due to the unique nature of the considered set up, the protocol had to be tailored to it, inspired by both mammography ${ }^{13}$ and $\mathrm{CT}^{14}$ protocols, and taking into account the coherent and monochromatic nature of the imaging beam. The protocol for the only existing commercial breast CT system ${ }^{15}$ was used as a starting point, including the structure of the dedicated phantom for image quality tests. For this purpose, a custom-made QC phantom was designed and built by the SYRMA-3D collaboration at the laboratories of the University of Ferrara, Italy. The present discussion will focus on the measurements regarding image quality, to be performed on a set of radiographic details embedded in the QC phantom, with the aim of validating the phantom as a viable tool for QC during the standard clinical practice.

\section{Materials and methods}

The radiation source of the SYRMEP beamline at Elettra is one of the storage ring bending magnets of the synchrotron machine. The beam is monochromatic in the energy range $8.5-40 \mathrm{keV}$ and the beam cross section in the patient examination room is about $220 \mathrm{~mm}$ (horizontal) $\times 3.4$ $\mathrm{mm}$ (vertical). The patient support was designed to perform breast $\mathrm{CT}$, by rotating the breast in a pendant geometry through an ergonomically designed aperture at the rotation axis. Concerning the tomographic set up of the SYRMA-3D project, the patient lays in prone position, with the breast hanging without compression from a hole in the patient support, placed at $30 \mathrm{~m}$ from the synchrotron source. Due to the peculiar laminar geometry of the beam, full breasts can only be imaged in steps equal to the vertical beam height, moving the patient support vertically after the acquisition of each slab is complete. The value of the air kerma rate is provided by a dosimetric system developed for the clinical mammography trial ${ }^{16}$ and based on two ionization chambers, placed $27 \mathrm{~m}$ downstream from the source and $3 \mathrm{~m}$ upstream from the breast. The dose monitor system of the beamline is based on two identical ionization chambers working in air, at atmospheric pressure. They were calibrated with respect to the air kerma primary standard chamber for low energy X-rays by the Department of Ionizing Radiation Metrology of ENEA (the National Agency for new Technologies, Energy and Sustainable Development). A check on the response of these monitors with respect to a calibrated secondary ionization chamber is performed annually. From the air kerma, the mean glandular dose (MGD) delivered to the scanned slice is evaluated from a custom-made Monte Carlo simulation based on a GEANT4 code optimized for breast dosimetry ${ }^{17,18}$, here assuming a 50\%-50\% breast composition. The images are acquired with PIXIRAD 8, a high efficiency, photon counting, direct conversion CdTe detector. ${ }^{19}$ This detector has a pixel size of $60 \mu \mathrm{m}$ and it is composed of 8 modules, for a global active area of $250 \mathrm{~mm} \times 25 \mathrm{~mm}$. The detec- 
tor is placed at about $2 \mathrm{~m}$ from the organ, in order to implement the free propagation phase-contrast technique.

The custom-made QC phantom was designed in such a way to mimic the geometrical appearance of a pendant human breast undergoing a CT examination, while including a set of details of radiographic interest for image quality tests. It is composed by a polymethyl methacrylate (PMMA) cylinder of $12 \mathrm{~cm}$ diameter and $10 \mathrm{~cm}$ height, filled with demineralized water to provide a uniform layer with attenuation properties similar to those of an actual breast. In particular, the cylinder diameter roughly corresponds to $13 \mathrm{~cm}$ of breast tissue in the energy range of interest. The uniform layer is useful to measure the uniformity of the CT reconstruction, and to ensure the absence of reconstruction artifacts. The phantom can be suspended from a PMMA disk of $35 \mathrm{~cm}$ diameter that is laid on the patient support, so as to hang it through the breast aperture, similarly to an actual breast CT examination. The depth of the suspension system can be adjusted to fit slight design modifications. This hanging configuration is depicted in Figure 1.

Several radiographic details are clung to the bottom of the cylinder: five rods of $1.2 \mathrm{~cm}$ diameter, made of different plastic materials, whose attenuation coefficients span the whole range of soft materials composing the breast; and a PMMA cylinder of $4.5 \mathrm{~cm}$ diameter, hereafter referred to as snail, with five holes of different diameters carved in it. The hole diameters span linearly the interval from $0.2 \mathrm{~cm}$ to $1.0 \mathrm{~cm}$. The height of both the rods and the snail is $3 \mathrm{~cm}$. The purpose of the plastic rods is to explore the linearity of the CT number reconstruction in the material range of interest: the reconstructed values are compared to the attenuation coefficients tabulated $\mathrm{in}^{20}$, in order to verify the linear relationship between them and to calibrate the conversion factor. The snail is designed to explore the device sensitivity to low contrast details: as the rest of the phantom, the holes are filled with water, whose attenuation properties are quite similar to those of PMMA in the selected energy range. The contrasts between the holes and the surrounding background serve as a measurement of the low contrast resolution of the imaging system. The distribution of the inserts is visible in Figure 1, which also lists the plastic materials composing the rods.

To summarize, the present version of the QC phantom allows to perform the following tests of physical image quality:

- CT number linearity;

- Accuracy of reconstructed attenuation coefficients;

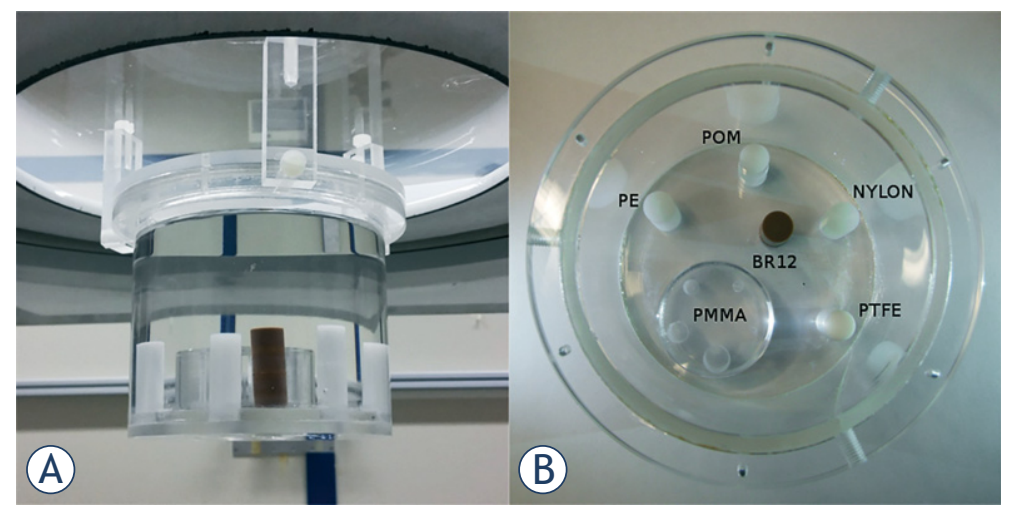

FIGURE 1. (A) QC phantom fixed at the breast position on the patient support. The upper portion is uniformly filled with water, while the radiographic details are visible in the lower portion. (B) Arrangement of the details at the bottom of the QC phantom. The rods are made of polyethylene (PE), nylon, polyoxymethylene (POM), polytetrafluoroethylene (PTFE), and BR12, a plastic material mimicking the attenuation properties of breast tissue. The structure of the low contrast PMMA insert, exhibiting the five holes of varying diameter, is clearly perceivable.

- Accuracy of the attenuation coefficient for water;

- Spatial uniformity in water;

- Noise fluctuations in water;

- Low contrast resolution.

Images were taken at four different energies, namely 32, 35, 38, and $40 \mathrm{keV}$. Each energy was imparted at two different dose levels, about $5 \mathrm{mGy}$ (low dose) and $20 \mathrm{mGy}$ (high dose). The phantom was scanned at two different heights, one in its upper portion and the other in its lower portion. As a result, two slabs of $3.4 \mathrm{~mm}$ thickness were reconstructed, the first reproducing a uniform layer of water, the second depicting the radiographic details. Regarding the former slab, only the central slice was considered in the analysis. On the contrary, an averaging over the ten central slices of the latter slab was performed. Clearly, due to the averaging procedure, the resulting image quality will not match the one of the actual diagnostic images. Nonetheless, the aim of the protocol is to provide reference values to be compared with the results of the periodic measurements. The choice of performing a slice average suppresses the relative contribution of stochastic fluctuations to the measure, thus enhancing the contribution of systematic effects that may decrease the image quality.

The images resulting from the reconstructions listed above will be hereafter referred to as water and detail images, respectively. All image quality tests are repeated on each one of the exposure conditions described above.

The images of the QC phantom were generated by rotating the phantom at a constant speed of 4.5 


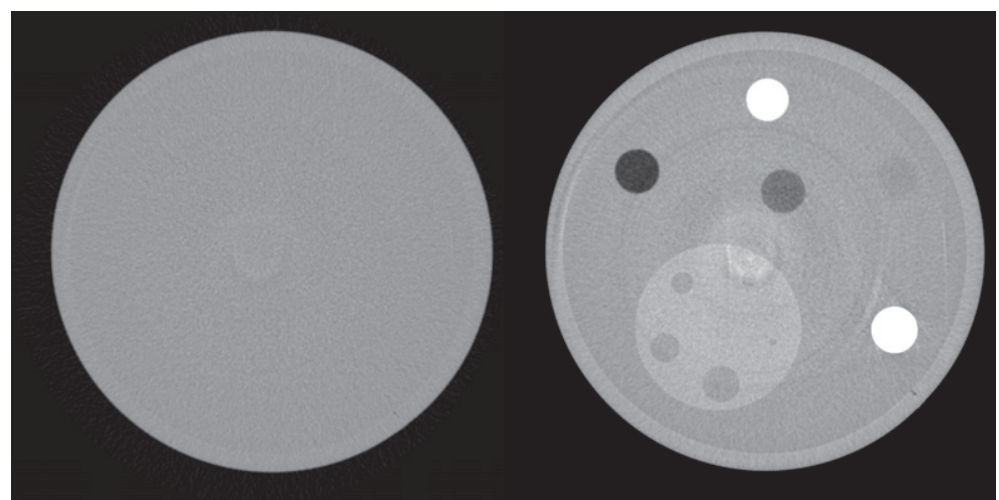

FIGURE 2. Example of reconstructed water image (left panel) and detail image (right panel), acquired at $38 \mathrm{keV}$ and high dose.

$\mathrm{deg} / \mathrm{s}$, acquiring 1200 projections over a $180^{\circ}$ angle, for a total exposure time of $40 \mathrm{~s}$. The frame rate of the acquisition was equal to 30 frame/s. The CT reconstruction was performed using a filtered backprojection algorithm with a Shepp-Logan filter.
The reconstructed image has a slightly anisotropic voxel of $60 \times 60 \times 52 \mu \mathrm{m}$ and is a 32-bit real, grayscale image. A subsequent $2 \times$ binning is applied to the image, with a resulting voxel of $120 \times 120 \times 104 \mu \mathrm{m}$. Finally, a smoothing filter is applied to the binned image. Exemplary reconstructed images, acquired at $38 \mathrm{keV}$ and high dose, are shown in Figure 2.

It should be noted that, due to the monochromaticity of the X-ray beam, the output of the CT reconstruction is a map of attenuation coefficients $\mu$. Therefore, it appears natural to express the results in terms of $\mu$ units, typically $\mathrm{cm}^{-1}$. However, the unit of measurement that is most widely used in clinical practice is the Hounsfield unit (HU), defined as

$H U=1000 \cdot \frac{\mu-\mu_{\text {water }}}{\mu_{\text {water }}-\mu_{\text {air }}}$.

This is the preferred definition for CT scanners that are calibrated with reference to water. Being the attenuation coefficient of air nearly zero, one $\mathrm{HU}$ corresponds to an attenuation coefficient that is $0.1 \%$ higher than the one of water.
CT Numbers Linearity $32 \mathrm{keV}$ High Dose

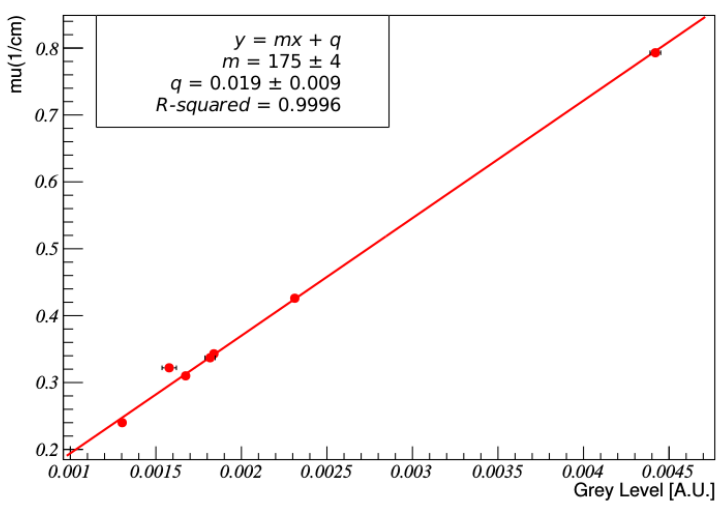

CT Numbers Linearity $38 \mathrm{keV}$ High Dose

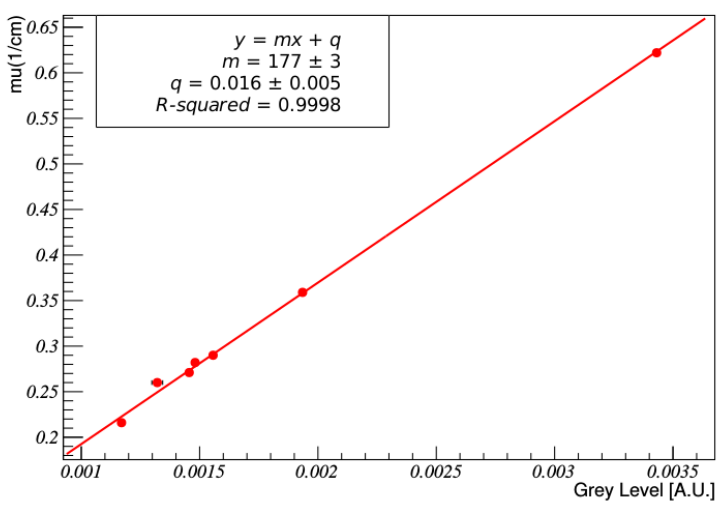

CT Numbers Linearity $35 \mathrm{keV}$ High Dose

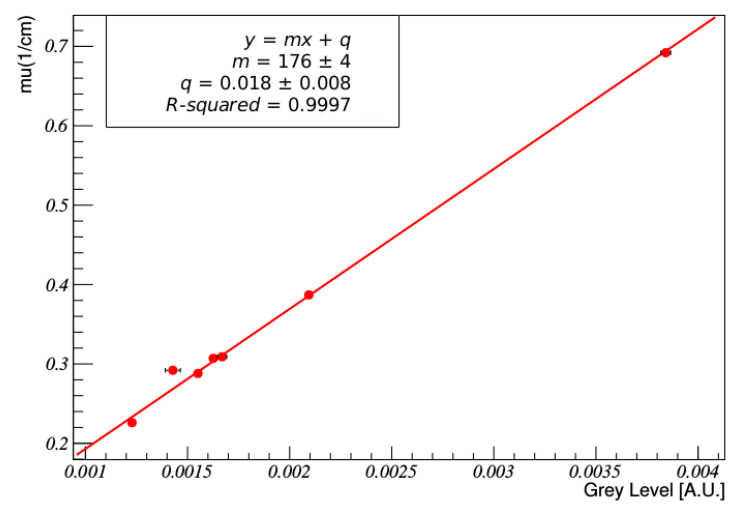

CT Numbers Linearity $40 \mathrm{keV}$ High Dose

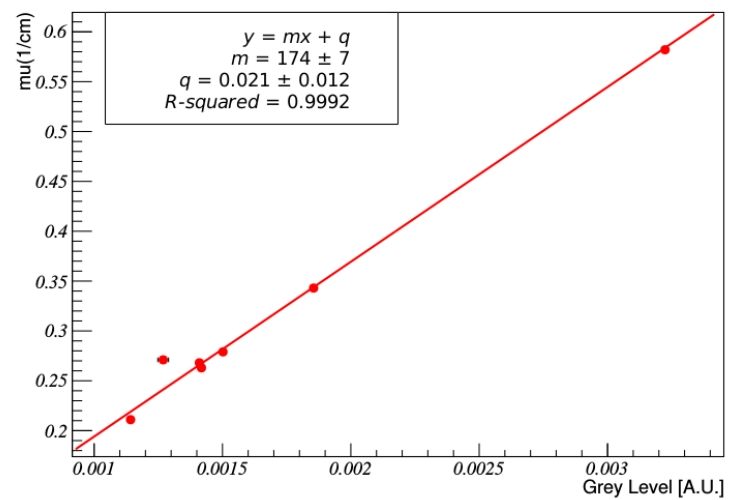

FIGURE 3. Examples of linear regressions, performed on high dose images acquired at 32, 35, 38 and $40 \mathrm{keV}$, from top left panel to bottom right panel. $\mathrm{R}^{2}$ coefficients, slopes and intercepts are given as well. 


\section{Results}

The first measurement to be performed is the CT number linearity. In the detail image, a circular region of about 80 pixel diameter is selected in the center of each rod. Average pixel values are computed, and compared to the tabulated attenuation coefficients. A linear fit allows to determine the CT number linearity (through the $\mathrm{R}^{2}$ coefficient), the conversion factor (slope of the regression line), and the absence of a nonzero threshold (comparability of the intercept with a zero value). The parameters of the linear regressions are given in Table 1, while some examples of linear regressions are shown in Figure 3. Both the fit parameters and the graphic representation demonstrate a good linearity for all energies.

Once the conversion factor is extracted, it is possible to evaluate the accuracy of the reconstructed attenuation coefficients of the plastic materials. Said accuracy is here defined as the relative percentual discrepancy between measured and expected values

$\operatorname{accuracy}_{\mu}=100 \cdot \frac{\left|\mu_{\text {meas }}-\mu_{\text {exp }}\right|}{\mu_{\text {exp }}}$.

Moreover, the HU deviation is also computed as the absolute difference between the corresponding HU values

accuracy $_{H U}=\left|H U_{\text {meas }}-H U_{\text {exp }}\right|$.

Exemplary results, taken at $38 \mathrm{keV}$ and high dose, are shown in Table 2. All accuracies lie within a few percent.

Accuracy must also be tested on water. This is performed on a set of three nonoverlapping regions in the center of the water image. Such regions are circular, with 80 pixel diameters. Averaged CT numbers are converted to a $\mu$ coefficient and compared with the expected value. As for the plastic materials, the result is given as a relative percentual difference. The HU deviation is also computed as before, the expected value in this case being identically zero. This particular measurement is the most natural candidate to be applied in a calibration procedure. Exemplary results, taken at 38 $\mathrm{keV}$ and high dose, are also given in Table 2.

As already said, the water image is supposed to be spatially uniform. However, reconstruction artifacts and X-ray beam disuniformities can lead to an uneven CT number distribution across different regions of the image. Therefore, an average is taken over a set of three nonoverlapping regions in the center of the water image, as for the accuracy measurement. Afterwards, equivalent measure-
TABLE 1. Parameters of the linear regressions between measured CT numbers and tabulated attenuation coefficients

\begin{tabular}{lcccc}
\hline Energy (keV) & Dose & $\mathbf{R}^{\mathbf{2}}$ & slope $\left(\mathrm{cm}^{-1}\right)$ & $\begin{array}{c}\text { intercept } \\
\left(\mathbf{c m}^{-1}\right)\end{array}$ \\
\hline 32 & high & 0.9996 & $175 \pm 4$ & $0.019 \pm 0.009$ \\
\hline 35 & low & 0.9996 & $170 \pm 4$ & $0.025 \pm 0.009$ \\
\hline 38 & high & 0.9997 & $176 \pm 4$ & $0.018 \pm 0.008$ \\
\hline 40 & low & 0.9996 & $173 \pm 4$ & $0.017 \pm 0.009$ \\
\hline & high & 0.9998 & $177 \pm 3$ & $0.016 \pm 0.005$ \\
\hline & low & 0.9992 & $175 \pm 3$ & $0.013 \pm 0.006$ \\
\hline
\end{tabular}

TABLE 2. Accuracies of reconstructed attenuation coefficients for the plastic materials and water, acquired at $38 \mathrm{keV}$ and high dose. Expected values, measured values and relative percentual differences are given for $\mu$ coefficients, absolute differences for the corresponding HU deviations

\begin{tabular}{ccccc}
\hline Material & $\begin{array}{c}\text { expected } \mu \\
\left(\mathbf{c m}^{-1}\right)\end{array}$ & $\begin{array}{c}\text { measured } \mu \\
\left(\mathbf{c m}^{-1}\right)\end{array}$ & $\begin{array}{c}\text { accuracy }_{\mu} \\
(\%)\end{array}$ & accuracy $_{\text {HU }}$ \\
\hline PE & 0.216 & $0.221 \pm 0.002$ & $3.1 \pm 1.3$ & $24 \pm 10$ \\
BR12 & 0.260 & $0.249 \pm 0.004$ & $4.0 \pm 1.6$ & $37 \pm 14$ \\
Nylon & 0.271 & $0.273 \pm 0.003$ & $0.9 \pm 1.0$ & $8 \pm 9$ \\
PMMA & 0.290 & $0.291 \pm 0.003$ & $0.4 \pm 1.2$ & $4 \pm 12$ \\
POM & 0.359 & $0.360 \pm 0.003$ & $0.2 \pm 0.8$ & $3 \pm 11$ \\
PTFE & 0.622 & $0.633 \pm 0.003$ & $0.1 \pm 0.5$ & $3 \pm 11$ \\
Water & 0.282 & $0.278 \pm 0.003$ & $1.5 \pm 0.9$ & $14 \pm 9$ \\
\hline
\end{tabular}

$\mathrm{BR} 12$ = breast-tissue equivalent material; $\mathrm{PE}=$ polyethylene; $\mathrm{PMMA}=$ polymethyl methacrylate; $\mathrm{POM}=$ polyoxymethylene PTFE $=$ polytetrafluoroethylene

TABLE 3. Uniformity of reconstructed attenuation coefficient for water. Relative percentual values are given for $\mu$ coefficients, absolute values for the corresponding $\mathrm{HU}$ deviations

\begin{tabular}{cccc}
\hline $\begin{array}{c}\text { Energy } \\
(\mathrm{keV})\end{array}$ & Dose & $\begin{array}{c}\text { uniformity } \\
\mathbf{( \% )}\end{array}$ & uniformity $_{\text {HU }}$ \\
\hline 32 & high & 1.79 & 17 \\
& low & 3.19 & 32 \\
35 & high & 1.45 & 12 \\
& low & 1.47 & 13 \\
& high & 2.58 & 26 \\
& low & 1.35 & 13 \\
& high & 2.54 & 24 \\
& low & 1.07 & 10 \\
\hline
\end{tabular}

ments are performed on similar regions close to the edge at the four cardinal points, and $\mu$ uniformity is defined as the largest absolute value among the relative percentual differences between the central and the peripheral averages 
TABLE 4. Noise measurement of the reconstructed attenuation coefficient for water. Relative percentual results are given for $\mu$ coefficients, absolute results for the corresponding HU values

\begin{tabular}{cccc}
\hline $\begin{array}{c}\text { Energy } \\
(\mathrm{keV})\end{array}$ & Dose & noise $_{\mu}(\%)$ & noise $_{\mathrm{HU}}$ \\
\hline 32 & high & 4.4 & 60 \\
& low & 8.5 & 103 \\
35 & high & 3.8 & 83 \\
\multirow{2}{*}{38} & low & 7.3 & 114 \\
& high & 3.7 & 66 \\
\multirow{2}{*}{40} & low & 6.5 & 109 \\
& high & 3.5 & 66 \\
& low & 6.2 & 111 \\
\hline
\end{tabular}

TABLE 5. Low contrast measurements. Relative percentual contrasts are given for $\mu$ coefficients, absolute contrasts for the corresponding Hounsfield unit (HU)

\begin{tabular}{|c|c|c|c|c|c|}
\hline \multirow{2}{*}{$\begin{array}{c}\text { Energy } \\
\text { (keV) }\end{array}$} & \multirow{2}{*}{$\begin{array}{l}\text { Detail } \\
(\mathrm{mm})\end{array}$} & \multicolumn{2}{|c|}{$\mu$ contrast (\%) } & \multicolumn{2}{|c|}{ HU contrast } \\
\hline & & high dose & low dose & high dose & low dose \\
\hline \multirow[t]{5}{*}{32} & 10 & 0.81 & 0.71 & 7 & 5 \\
\hline & 8 & 0.96 & 0.87 & 9 & 7 \\
\hline & 6 & 1.10 & 0.53 & 10 & 4 \\
\hline & 4 & 1.08 & 1.12 & 10 & 10 \\
\hline & 2 & 0.52 & 0.93 & 5 & 8 \\
\hline \multirow[t]{5}{*}{35} & 10 & 1.80 & 1.85 & 15 & 15 \\
\hline & 8 & 1.68 & 1.73 & 14 & 14 \\
\hline & 6 & 1.56 & 1.64 & 13 & 13 \\
\hline & 4 & 1.21 & 0.87 & 10 & 8 \\
\hline & 2 & 0.90 & 0.91 & 8 & 8 \\
\hline \multirow[t]{5}{*}{38} & 10 & 4.14 & 3.86 & 41 & 39 \\
\hline & 8 & 3.99 & 3.41 & 40 & 34 \\
\hline & 6 & 3.66 & 4.02 & 36 & 41 \\
\hline & 4 & 3.91 & 3.45 & 39 & 35 \\
\hline & 2 & 3.17 & 3.79 & 31 & 38 \\
\hline \multirow[t]{5}{*}{40} & 10 & 5.35 & 5.49 & 54 & 55 \\
\hline & 8 & 5.09 & 5.43 & 52 & 55 \\
\hline & 6 & 5.12 & 4.85 & 52 & 49 \\
\hline & 4 & 5.31 & 3.41 & 54 & 34 \\
\hline & 2 & 5.73 & 3.79 & 59 & 38 \\
\hline
\end{tabular}

uniformity $_{\mu}=100 \cdot \max \left\{\frac{\left|\mu_{\text {center }}-\mu_{i}\right|}{\mu_{\text {center }}}\right\}$

$i=$ north, west, south, east.

Alongside the previous measurements, the absolute difference between the corresponding $\mathrm{HU}$ values is measured as well uniformity $_{H U}=\max \left\{\left|H U_{\text {center }}-H U_{i}\right|\right\}$

$i=$ north, west, south, east.

Results are given in Table 3, showing that uniformity lies within a few percent for all energies.

Even in the case of a globally uniform water image, image quality could be spoiled by local fluctuations. As a result, a noise measurement is also necessary. Standard deviations $\sigma$ are computed over the three central regions defined in the accuracy measurement, and then averaged. The corresponding percentual $\mu$ noise is defined as the ratio between such $\sigma$ average and the globally averaged $\mu$ coefficient

noise $_{\mu}=100 \cdot \frac{\sigma_{\mu}}{\mu_{\text {averaged }}}$,

while the HU noise is defined as the average of the three standard deviations expressed in $\mathrm{HU}$

noise $_{H U}=\frac{\sigma_{H U}}{H U_{\text {averaged }}}$.

Results are given in Table 4. Noise values are distributed around an average of about $5 \%$.

The last image quality assessment is performed on the low contrast details of the snail. First, CT number averages are computed over five regions of interest, strictly included in the corresponding holes (concentric to the holes and $4 / 5$ of the hole diameters). These are used as signals. Then, CT number averages and standard deviations are computed over five annuli, each concentric to a hole and with inner and outer diameters of $6 / 5$ and $7 / 5$ of the hole diameter, respectively. These are used instead as backgrounds. The contrasts between the signals and the backgrounds, percentual relative contrasts for the $\mu$ coefficients and absolute contrasts for the corresponding HU values, are given in Table 5. The numerical values exhibited by the contrasts are low, yet in agreement with the expected ones.

\section{Discussion}

As already stated, the main goal of the present analysis is to assess the viability of the phantom as a QC tool for the upcoming breast CT imaging device. Nonetheless, secondary evaluations related to the quality of the CT reconstruction and the exposure parameters are possible as well.

Starting from the CT number linearity measurements, the plastic materials were selected in such a way to span the range of attenuation coefficients typical of a breast (both healthy and tumor tissues), thus guaranteeing the linearity of the re- 
construction within the attenuation range of breast tissues. As long as the linearity is guaranteed in that range, and the noise is kept below acceptable levels, the reconstruction will be able to capture both tissue contrasts and morphological features. It can be seen from Table 1 that all regressions exhibit a very high linearity $\left(1-R^{2}\right.$ is always less than $\left.10^{-3}\right)$, while the slopes are extremely stable against exposure parameters variations. All slopes are compatible with each other within uncertainty intervals, as reported in Table 1. A slight systematic offset is noticeable in the intercept values, probably due to the data point corresponding to the breast-tissue equivalent BR12 material, whose chemical composition (and therefore attenuation coefficient) is known with lower accuracy than the other materials. Weighted average values for intercept and slope are $0.018 \pm 0.003 \mathrm{~cm}^{-1}$ and $174.4 \pm 1.6$ $\mathrm{cm}^{-1}$, respectively. Such linearity assessment represents a validation of the reconstruction algorithm, a quality check that will prove particularly useful if the filtered back-projection is replaced by more complex algorithms.

CT number accuracy is another important quality of a CT imaging system. Table 2 shows that the accuracies are comparable with zero within two standard deviations. Measurements at other exposure conditions exhibit similar accuracies. Such result guarantees that the soft tissues composing the imaged breasts will be correctly reproduced by the imaging system, and that the measurement provides a stable reference for $\mathrm{CT}$ number calibration.

Regarding the uniformity of the water image, it can be seen from Table 3 that disuniformities lie within few percent for all exposure conditions. The water image noise measurements give slightly higher values, probably due to the voxel size, which is smaller than the typical CT system. Moreover, it can be seen that noise values approximately double from high to low doses, as one would expect from the behavior of stochastic fluctuations.

Finally, low contrast details analysis allows to draw two important conclusions. First of all, although the $\mu$ contrasts are comparable with the expected ones, as one would assume from an accurate CT imaging system, it is clear from Table 5 that some of the corresponding HU contrasts do not match the ones typically suggested by QC protocols for CT, e.g. Koning..$^{15}$ The main issue here is the composition of the snail: the attenuation coefficients of PMMA and water are too similar in the selected energy range to provide a reliable reference, especially for what regards the lower portion of the range. Therefore, it will either be necessary to fill the holes with a material other than water, or to change the composition of the snail, or to select another energy range for the examinations. However, since the optimal imaging energy is mainly determined by the quality of the $\mathrm{CT}$ reconstruction, the final choice of materials for the snail will stem from an energy optimization procedure that is still ongoing.

There is one last point worth mentioning, concerning the detectability assessment of high contrast details. A rod made of a more attenuating material like polyvinyl chloride (PVC), originally included in the phantom to mimic the attenuation properties of microcalcifications, was discarded to be replaced by a high contrast insert. This is because such an isolated data point would skew the output of the linear regression, partially hiding the information about the actual linearity in the soft tissues range. On the other hand, linearity is a much looser requirement in the imaging of small details like microcalcifications. This high contrast insert is not included in the present version of the QC phantom, but will be present in the final version. A $2 \mathrm{~cm}$ thick disk, supposedly made of synthetic resin, will be suspended in the upper portion of the phantom, above the plastic inserts. Such disk will contain several clusters of specks made of a highly absorbing material, mimicking the attenuation properties of microcalcifications, and a straight tungsten wire tilted at a given angle with respect to the vertical direction. Such details will allow measurements of the system response to small, high contrast details, of its modulation transfer function and of the thickness of the reconstructed slice. These measures will become part of the QC protocol.

Having reviewed in detail the results of each measurement, there are a few general remarks that are worth making. First of all, with the sole exception of the low contrast one, the phantom inserts proved able to allow precise image quality measurements. Moreover, the morphology of the phantom turned out adequate to fit the geometrical set up of the examination. Therefore, once complemented with the high contrast insert and the upgraded snail, the QC phantom will be ready to be used as a QC tool in standard clinical practice. Finally, it is worth stressing that the measurement procedures included in the final version of the protocol will be complemented with the corresponding reference values obtained in optimal experimental conditions, which are essential components of a QC protocol. 


\section{Acknowledgements}

The SYRMA-3D project is funded by INFN (the National Institute for Nuclear Physics), and we wish to thank all the members of the SYRMA-3D collaboration. Author SD was partially funded by "Fondazione per la Fisica - Trieste".

\section{References}

1. Chang CH, Sibala JL, Fritz SL, Gallagher JH, Dwyer 3rd SJ, Templeton AW. Computed tomographic evaluation of the breast. Am J Roentgenol 1978; 131: 459-64. doi: 10.2214/ajr.131.3.459

2. Boone JM, Nelson TR, Lindfors KK, Seibert JA. Dedicated breast CT: radiation dose and image quality evaluation. Radio/ 2001; 221: 657-67. doi: 10.1148/ radiol.2213010334

3. Kalender, WA, Kolditz D, Steiding C, Ruth V, Lück F, Rößler AC, et al. Technical feasibility proof for high-resolution low-dose photon-counting CT of the breast. Eur Radiol 2017; 27: 1081-6. doi: 10.1007/s00330-016-4459-3

4. New York Koning Corporation, West Henrietta, NY. Koning breast CT. [cited 2017 Aug 14]. Available at http://koninghealth.com/en/kbct

5. Longo R, Arfelli F, Bellazzini R, Bottigli U, Brez A, Brun F, et al. Towards breast tomography with synchrotron radiation at Elettra: first images. Phys Med Biol 2016; 61: 1634-49. doi: 10.1088/0031-9155/61/4/1634

6. Sarno A, Mettivier G, Golosio B, Oliva P, Spandre G, Di Lillo F, et al. Imaging performance of phase-contrast breast computed tomography with synchrotron radiation and a CdTe photon-counting detector. Phys Med 2016; 32: 681-90. doi: 10.1016/j.ejmp.2016.04.011

7. Delogu P, Oliva P, Bellazzini R, Brez A, De Ruvo PL, Minuti M, et al. Characterization of Pixirad-1 photon counting detector for X-ray imaging. $J$ Instrum 2016; 11: P01015. doi: 10.1088/1748-0221/11/01/P01015

8. Longo R. Current studies and future perspectives of synchrotron radiation imaging trials in human patients. Nucl Instr Meth Phys Res A 2016; 809: 13 22. doi: 10.1016/j.nima.2015.10.110A

9. Rigon L. x-Ray Imaging with Coherent Sources. In: Brahme A, editor. Comprehensive Biomedical Physics. Vol. 2. Amsterdam: Elsevier; 2014. p. $193-220$.

10. Moeckli R, Verdun FR, Fiedler S, Pachoud M, Schnyder P, Valley JF. Objective comparison of image quality and dose between conventional and synchrotron radiation mammography. Phys Med Biol 2000; 45: 3509-23. doi: 10.1088/0031-9155/45/12/301

11. Baldelli P, Taibi A, Tuffanelli A, Gambaccini M. Dose comparison between conventional and quasi-monochromatic systems for diagnostic radiology. Phys Med Biol 2004; 49: 4135-46. doi: 10.1088/0031-9155/49/17/021

12. Veronese A. A proposal for a quality control protocol in breast CT with synchrotron radiation. [Master Thesis]. Trieste: Universita degli Studi di Trieste, Dipartimento di Fisica; 2017.

13. European Reference Organization for Quality Assured Breast Screening EUREF and Diagnostic Services. European protocol for the quality control of the physical and technical aspects of mammography screening. In: European guidelines for quality assurance in breast cancer screening and diagnosis. Fourth edition. Perry N, Broeders $\mathrm{M}$, de Wolf C, Törnberg S, Holland R, von Karsa L, editors. Luxembourg: Office for Official Publications of the European Communities; 2006. p. 57-166

14. Edyvean S, Jones A. Computed tomography x-ray scanners. In: Measurement of the performance characteristics of the diagnostic $x$-ray systems used in medicine. IPEM Report n. 32 Part III. York: IPEM; 2003.

15. Koning. User's manual for Koning breast CT system. West Henrietta, New York: Koning Corporation. [cited 2017 Aug 14]. Available at www.accessdata.fda.gov/cdrh docs/pdf13/P130025c.pdf

16. Castelli E, Tonutti M, Arfelli F, Longo R, Quaia E, Rigon L, et al. Mammography with synchrotron radiation: first clinical experience with phase-detection technique. Radiol 2011; 259: 684-94. doi: 10.1148/radiol.11100745
17. Fedon C, Longo F, Mettivier G, Longo R. GEANT4 for breast dosimetry: parameters optimization study. Phys Med Biol 2015; 60: 311-23. doi: 10.1088/0031-9155/60/16/N311

18. Mettivier G, Fedon C, Di Lillo F, Longo R, Sarno A, Tromba G, et al. Glandular dose in breast computed tomography with synchrotron radiation. Phys Med Biol 2016; 61: 569-87. doi: 10.1088/0031-9155/61/2/569

19. Bellazzini R, Spandre G, Brez A, Minuti M, Pinchera M, Mozzo P. Chromatic $X$-ray imaging with a fine pitch CdTe sensor coupled to a large area photon counting pixel ASIC. J Instrum 2013; 8: C02028. doi: 10.1088/17480221/8/02/C02028

20. Boone JM, Chavez AE. Comparison of $x$-ray cross sections for diagnostic and therapeutic medical physics. Med Phys 1996; 23: 1997-2005. doi:10.1118/1.597899 